\title{
Peripheral and local predictive immune signatures identified in a phase II trial of ipilimumab with carboplatin/paclitaxel in unresectable stage III or stage IV melanoma
}

Rahima Jamal ${ }^{1}$, Réjean Lapointe ${ }^{2}$, Eftihia Cocolakis ${ }^{3}$, Paméla Thébault ${ }^{2}$, Shirin Kazemi ${ }^{3}$, Jennifer E. Friedmann ${ }^{3}$, Jeanne Dionne ${ }^{1}$, Jean-François Cailhier ${ }^{2}$, Karl Bélanger ${ }^{1}$, Jean-Pierre Ayoub ${ }^{1}$, Huy Le $^{3}$, Caroline Lambert ${ }^{3}$, Jida El-Hajjar ${ }^{3}$, Léon C. van Kempen ${ }^{4}$, Alan Spatz ${ }^{4}$ and Wilson H. Miller $\mathrm{Jr}^{3^{*}}$

\begin{abstract}
Background: Checkpoint blockade with ipilimumab provides long-term survival to a significant proportion of patients with metastatic melanoma. New approaches to increase survival and to predict which patients will benefit from treatment are needed. This phase II trial combined ipilimumab with carboplatin/paclitaxel (CP) to assess its safety, efficacy, and to search for peripheral and tumor-based predictive biomarkers.

Methods: Thirty patients with untreated unresectable/metastatic melanoma were treated with ipilimumab and CP. Adverse events (AEs) were monitored and response to treatment was evaluated. Tumor tissue and peripheral blood were collected at specified time points to characterize tumor immune markers by immunohistochemistry and systemic immune activity by multiplex assays and flow cytometry.

Results: Eighty three percent of patients received all 5 cycles of CP and 93\% completed ipilimumab induction. Serious AEs occurred in 13\% of patients, and no treatment-related deaths were observed. Best Overall Response Rate (BORR) and Disease Control Rate (DCR) were 27 and 57\%, respectively. Median overall survival was 16. 2 months. Response to treatment was positively correlated with a higher tumor $\mathrm{CD}^{+}$infiltrate (immune score) at baseline. NRAS and BRAF mutations were less frequent in patients who experienced clinical benefit. Assessment of peripheral blood revealed that non-responders had elevated baseline levels of CXCL8 and CCL4, and a higher proportion of circulating late differentiated B cells. Pre-existing high levels of chemokines (CCL3, CCL4 and CXCL8) and advanced B cell differentiation were strongly associated with worse patient overall survival. Elevated proportions of circulating $\mathrm{CD} 8^{+} / \mathrm{PD}-1^{+} \mathrm{T}$ cells during treatment were associated with worse survival.

Conclusions: The combination of ipilimumab and CP was well tolerated and revealed novel characteristics associated with patients likely to benefit from treatment. A pre-existing systemic inflammatory state characterized by elevation of selected chemokines and advanced B cell differentiation, was strongly associated with poor patient outcomes, revealing potential predictive circulating biomarkers.
\end{abstract}

Trial registration: Clinicaltrials.gov, NCT01676649, registered on August 29, 2012.

Keywords: Melanoma, Ipilimumab, Chemotherapy, Chemokines, Immune score, B lymphocytes, PD-1, Predictive Biomarkers, Immunotherapy

\footnotetext{
*Correspondence: wilsonmiller@gmail.com

${ }^{3}$ Segal Cancer Center, Jewish General Hospital, Rossy Cancer Network, McGill

University, 3755 Côte-St-Catherine, suite E670, Montreal, Québec, Canada

Full list of author information is available at the end of the article
} 


\section{Background}

Metastatic melanoma has been historically one of the most treatment-resistant human malignancies, with a median survival of 6-9 months with standard chemotherapy [1]. Recently, major strides in the treatment of advanced melanoma were achieved with the approval of two classes of therapies, targeting the MAPK pathway and immune checkpoints. Ipilimumab, a fully humanized monoclonal antibody targeting cytotoxic T-lymphocyte-associated protein 4 (CTLA-4) and a negative regulator of T-cell activation, was the first immunotherapeutic agent shown to prolong survival in this disease [2, 3]. Pivotal studies with ipilimumab demonstrated increased overall survival (OS) as a single agent (10.1 months) and in combination with DTIC (11.2 months). Combining ipilimumab with other treatment modalities to increase its efficacy and searching for predictive biomarkers have been areas of intense research [4-9]. After failure of immune checkpoint inhibitors or in patients progressing rapidly after BRAF and MEK inhibitors, chemotherapies, such as carboplatin and paclitaxel $(\mathrm{CP})$, are still used to treat rapidly growing metastatic melanoma.

Previous studies have demonstrated a role for chemotherapeutic agents in promoting anti-tumor immunity through several mechanisms. Platinum compounds have been found to induce immunogenic cell death [10, 11], a functionally distinct type of apoptosis that elicits tumorspecific cognate immune responses [12]. Preclinical studies show tumor cells treated with taxanes or platinum compounds lead to increased cytotoxic T-cell activation [13-15]. Paclitaxel has also been implicated in direct inhibition of myeloid-derived suppressor cells (MDSCs), a heterogeneous population of cells that are defined by their myeloid origin, immature state and ability to potently suppress $\mathrm{T}$ cell responses [16]. Addition of chemotherapy has been shown to enhance antiCTLA-4 antitumor activity in animal models [17]. Thus, there is a good rationale to combine immune checkpoint inhibitors, such as ipilimumab, with platinum compounds like carboplatin and taxanes such as paclitaxel.

Safety and anti-tumor efficacy of this combination was evaluated. This study also sought to prospectively identify cellular and/or molecular predictive biomarkers.

\section{Methods}

\section{Study design and treatment}

Eligible patients were men and women $\geq 18$ years with a histologic diagnosis of untreated, unresectable Stage III/ Stage IV melanoma. Prior treatment with BRAF inhibitors in the metastatic setting was permitted. Patients had measurable or evaluable disease, Eastern Cooperative Oncology Group (ECOG) performance status $\leq 1$ and adequate bone marrow, liver, and renal function at study entry. Patients with symptomatic brain metastases, autoimmune disease, peripheral neuropathy $\geq$ Grade 2, prior treatment with a CD137 agonist or a CTLA-4 agonist/inhibitor, chronic use of immunosuppressive drugs or of systemic corticosteroids were excluded.

Treatment consisted of carboplatin (AUC 6)/paclitaxel $\left(175 \mathrm{mg} / \mathrm{m}^{2}\right) \times 5$ cycles and ipilimumab $(3 \mathrm{mg} / \mathrm{kg} \times 4 \mathrm{cy}-$ cles) every 3 weeks. Each CP treatment was preceded by dexamethasone premedication. Two different dosing schedules were used, patients were randomly assigned in a 1:2 scheme to receive ipilimumab either concurrently (Arm A, 10 patients) with CP or sequentially 1 week apart (Arm B, 20 patients), starting at Cycle 2. Patients were stratified according to previous treatment with a BRAF inhibitor. Physicians had the option of retreating patients with either ipilimumab alone or in combination with CP if they experienced disease progression following objective response (PR, CR) or SD lasting for 3 months or more. Supportive measures and/or secondary prophylaxis were allowed for CP induced hematologic toxicity.

The primary objective was to determine the safety and tolerability of two schedules of ipilimumab in combination with CP. Secondary objectives were to determine putative early cellular and/or molecular biomarkers for therapy response; to measure anti-tumor efficacy (OS, overall response rate (ORR), PFS, and clinical benefit rate $(\mathrm{CBR}$; ORR + stable disease $(\mathrm{SD}) \geq 24$ weeks)) by irRC and $\mathrm{mWHO}$ response criteria [18].

Response evaluations (progressive disease (PD), stable disease $(\mathrm{SD})$, partial response $(\mathrm{PR})$, complete response (CR)) were defined according to $\mathrm{mWHO}$ and irRC guidelines [18]. mWHO results are available in Additional file 1: Figure S1 and Additional file 2: Table S3. Tumor assessments were performed at screening, weeks 8, 16, 24 and approximately every 12 weeks thereafter. Safety assessments included vital signs, physical examinations, laboratory tests, and adverse event reporting (AEs). AEs were graded using the Common Toxicity Criteria for Adverse Events, version 4.0. AEs were recorded from the first study medication dose. SAEs were recorded following consent signature until 70 days from discontinuation of investigational products.

The study was conducted in accordance with the Declaration of Helsinki of 1975. The protocol was approved by local ethics committees. All participants gave written informed consent for the study.

\section{Correlative studies}

DNA was extracted from formalin-fixed and paraffinembedded tissue sections containing at least 30\% tumor cells (cobas ${ }^{\oplus}$, Roche) collected at screening. Mutation analysis of BRAF V600 (cobas ${ }^{\oplus}$ kits, Roche) was performed on a $\operatorname{cobas}^{\oplus} \mathrm{z} 480$ instrument and confirmed by sequencing (Sanger). For NRAS, mutation analysis was performed using the NRAS Mutation detection kit 
(EntroGen) on an LC480 platform (Roche). All manipulations were performed according to College of American Pathologists-compliant standard operating procedures of the Jewish General Hospital Molecular Pathology Centre.

Immunohistochemistry with Ventana Benchmark antibodies towards CD3 (clone 2GV6), CD4 (clone SP35), CD8 (clone 1A5) and PD-L1 (clone SP142) was performed according to standard automated protocols and quantified in agreement with REMARK guidelines [19]. PD-L1 expression was assessed semi-quantitatively as previously described [20]. The immune score was obtained from semi-quantitative prevalence of $\mathrm{CD}^{+}$cells noted as absent (0), focal (1), moderate (2) or severe (3) as previously described [21].

Blood samples were collected from patients at baseline, weeks 1, 2, 4, 7, 10,13,16, 24 and at the end of study. Blood was also obtained from six normal donors (ND). Peripheral blood collection and processing were done as previously described [22]. PBMCs suspended in human serum albumin solution were stored at $-80{ }^{\circ} \mathrm{C}$. Cell surface staining was performed as described previously $[22,23]$ using antibodies (BD Biosciences) listed in Additional file 2: Table S6. Dead cells were excluded with the Live/Dead Fixable Dead Cell kit (Invitrogen). Flow cytometry data were acquired using a BDFortessa instrument (BD Biosciences) and analyzed using FlowJo software (Tree Star). Peripheral soluble cytokines/chemokines/soluble receptors (Additional file 2: Table S7) were measured from cryopreserved plasma. All kit components from V-plex Ultra-Sensitive kit (Meso Scale Discovery) were processed as per the manufacturer's instructions. Electroluminescent data were analyzed with a four-parameter logistic curve fit using MSD Discovery Workbench.

IgG, IgA and IgM concentrations were determined in a standard ELISA as previously previously described [14]. Baseline values from patients and from the normal donors were inside the accepted ranges of reference values for clinical diagnostic purposes (total IgG, 7.0-16.0 g/l; total IgA, 0.7-4.0 g/l; total IgM, 0.4-2.3 g/l).

\section{Statistical analyses}

This study was designed as a pilot study on a limited number of patients to gain insight on safety and efficacy of the combination of ipilimumab and $\mathrm{CP}$ and identify putative biomarkers associated with response. Patients and disease characteristics were analyzed using descriptive statistics and expressed as either relative frequency (percentages) for discrete variables or median for continuous variables. Comparisons between patient response groups at baseline and throughout treatment were evaluated by ANOVA. Correlations between mutation and tumor immune profiles and best overall response (BOR) were determined using Chi-square and
Pearson correlation tests. Effect of groups and time points and their interaction in blood samples were assessed with linear mixed effect models, multiple comparisons specified in figure legends were done where interactions were not significant. Univariate cox proportional hazards regression analyses were used to assess correlation of variables with OS. Chemokine, $\mathrm{Bm} 2$ and eBm $5+\mathrm{Bm} 5$ levels were logarithmically transformed prior to regression analyses. OS Kaplan-Meier curves were compared by the log-rank test. Cutoff thresholds defining high or low levels were established from the mean value of a given variable from all patients. A $P$ value $<0.05$ was considered statistically significant. Statistical analyses were performed with SPSS 25 software (SPSS Inc.), GraphPad Prism 6.01, and R software i386 3.0.2 [24].

\section{Results}

\section{Patient selection}

Thirty patients were enrolled onto the study from December 2012 until October 2013 (Table 1, Additional file 2: Table S1). Fifty-seven percent of patients had M1c disease, and 33\% of patients had LDH levels higher than the upper limit of normal at baseline. Seventeen percent of patients had received prior adjuvant therapy. Nine patients had a BRAF mutated melanoma, of whom six had been treated with a BRAF inhibitor in the metastatic setting. Eighty-three percent of patients received all five cycles of CP, and 93\% received all four doses of ipilimumab. One patient did not receive ipilimumab because of early clinical progression. Six patients meeting the retreatment criteria were retreated with either ipilimumab/CP $(2 / 6)$ or ipilimumab alone (4/6).

\section{Toxicity}

The most common Grade 3-4 adverse events found in $\geq 10 \%$ of patients were diarrhea, neutropenia and thrombocytopenia (Table 2, Additional file 2: Table S2). The rate of febrile neutropenia was 7\% (2/30). Four patients $(13 \%)$ had treatment-related SAEs. Two patients discontinued chemotherapy because of drug-related AEs (Grade 3 ALT and Grade 4 neutropenia). Four patients had infusion related reactions to paclitaxel. Grade 3-4 AEs related to ipilimumab were found in $13 \%$ of patients. Twenty percent (6/30) of patients received steroids for immune-related adverse events (irAEs): one patient with Grade 3 autoimmune colitis, two patients with Grade 3 diarrhea, two patients with Grade 2 endocrinopathy, and one patient with Grade 2 rash. One patient discontinued ipilimumab because of an irAE (Grade 3 autoimmune colitis) during the re-treatment period. No drug-related deaths were reported. Adverse events were comparable between treatment arms (Additional file 2: Table S2). 
Table 1 Demographic and baseline characteristics of the patients

\begin{tabular}{|c|c|}
\hline Patient Characteristics & $\begin{array}{l}\text { ALL patients } \\
\text { (Number (percent }\end{array}$ \\
\hline Total no. of patients & $30(100)$ \\
\hline Median age, years (range) & $55(26-74)$ \\
\hline \multicolumn{2}{|l|}{ Sex } \\
\hline Female & $8(27)$ \\
\hline Male & $22(73)$ \\
\hline \multicolumn{2}{|l|}{ Metastatic stage (n) } \\
\hline MO & $1(3)$ \\
\hline M1a & $6(20)$ \\
\hline $\mathrm{M} 1 \mathrm{~b}$ & $6(20)$ \\
\hline M1c & $17(57)$ \\
\hline \multicolumn{2}{|l|}{ Lactate dehydrogenase } \\
\hline$\leq \operatorname{ULN}^{a}$ & $20(67)$ \\
\hline$>$ ULN & $10(33)$ \\
\hline \multicolumn{2}{|l|}{ ECOG } \\
\hline 0 & $19(63)$ \\
\hline 1 & $11(37)$ \\
\hline \multicolumn{2}{|l|}{ Primary site } \\
\hline Cutaneous & $24(80)$ \\
\hline Mucosal & $2(7)$ \\
\hline Ocular & $3(10)$ \\
\hline Unknown primary & $1(3)$ \\
\hline \multicolumn{2}{|l|}{ BRAF status } \\
\hline BRAF mutated (V600E) & $9(30)$ \\
\hline BRAF wild type & $21(70)$ \\
\hline \multicolumn{2}{|l|}{ Prior therapies ${ }^{* *}$} \\
\hline Prior adjuvant therapy & $5(17)$ \\
\hline Prior therapy with a BRAF inhibitor & $6(20)$ \\
\hline \multicolumn{2}{|l|}{ Brain metastases } \\
\hline Patients without brain metastases & $26(87)$ \\
\hline Patients with brain metastases & $4(13)$ \\
\hline
\end{tabular}

ULN denotes upper limit of the normal, ** One patient (3\%) received 2 weeks a of Temozolomide

\section{Efficacy}

Patients were followed for up to 46.9 months, with an overall median follow-up of 37.6 months. The median OS was 16.2 months for the entire cohort (Figure 1a). The rate of OS for all patients was $56.7 \%, 43.3 \%$ and $36.7 \%$ at 1,2 and 3 years, respectively. The median PFS was 5.8 months by irRC for all patients (Figure 1b). The best overall response rate (BORR; $C R+P R)$, rate of disease control (DCR; $C R+P R+S D)$, and clinical benefit rate (CBR; $\mathrm{CR}, \mathrm{PR}$ or $\mathrm{SD} \geq 24$ weeks) for 30 evaluable patients were $27 \%, 57 \%$ and $43 \%$ by irRC, respectively (Table 3). The two mucosal patients had a BOR of PR whereas the three ocular patients all had PD.
Table 2 Adverse events

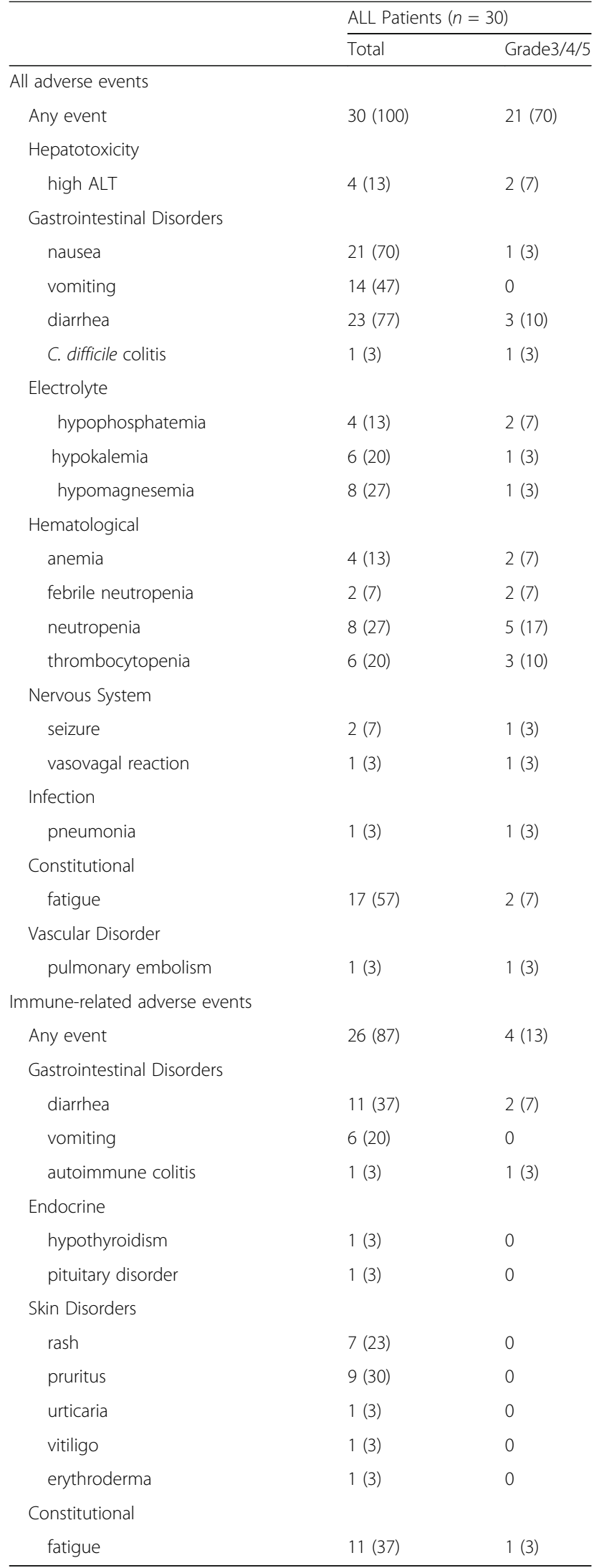




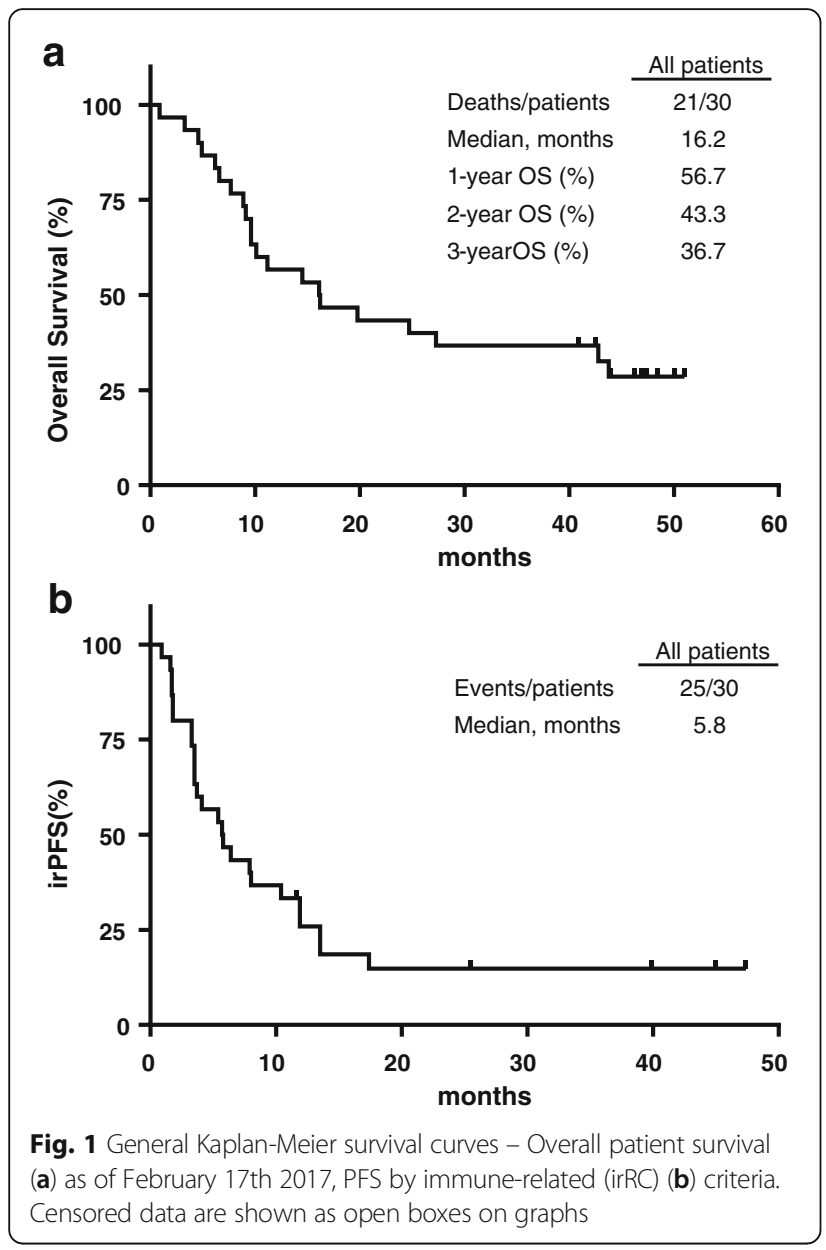

Tumor correlative studies

Prevalence of $B R A F$ and NRAS mutations, immune infiltration and PD-L1 expression in the tumor samples obtained pre-treatment were classified according to response. BRAF, NRAS and concurrent BRAF/

Table 3 Tumor response (immune related - irRC)

\begin{tabular}{|c|c|}
\hline \multirow[t]{3}{*}{ Response } & All patients \\
\hline & $n=30$ \\
\hline & No. of pts. (\%) \\
\hline \multicolumn{2}{|l|}{ irBOR } \\
\hline $\operatorname{irCR}$ & $1(3)$ \\
\hline irPR & $7(23)$ \\
\hline irSD & $9(30)$ \\
\hline irPD & $13(43)$ \\
\hline $\mathrm{irDCR}$ & $17(57)$ \\
\hline $\operatorname{irCBR}$ & $13(43)$ \\
\hline irBORR & $8(27)$ \\
\hline
\end{tabular}

NRAS mutations in pre-treatment samples were found in 8/30 (27\%, all V600E), 2/30 (7\%, both Q61R) and $1 / 30$ (3\%, V600E/Q61R) cases, respectively. Mutations in these genes were more frequently observed in PD patients $(9 / 17)$ compared to the patients that achieved clinical benefit $(2 / 13, p=0.034$, Chi-square 4.474, Figure 2a). No correlation between $B R A F$ and/or $N R A S$ mutation status and OS was observed. Baseline immune score reflecting prevalence of $\mathrm{CD}^{+}$cells in tumor samples directly correlated with BORR (Pearson correlation 0.487, $p=0.01$, Figure 2b). Immunohistochemistry analysis of PD-L1 expression at baseline was performed on 22 cases. Twenty-seven percent of cases (6/22) harbored greater than $5 \%$ of PD-L1 positive melanoma cells. Lack of PD-L1 expression $(<5 \%)$ was most frequently observed in irPD (10/12 samples (83\%) were PD-L1 negative) compared to SD (1/4 samples (25\%) and PR + CR (3/6 samples (50\%) were PD-L1 negative) but this did not reach statistical significance (Additional file 2: Table S4). No correlation between the percentage of $\mathrm{PD}-\mathrm{L} 1$ positive melanoma cells, immune score or BOR or OS was found (Figure 2c, Additional file 2: Table S4, Additional file 2: Table S5).

\section{Peripheral blood correlative studies: pre-treatment inflammatory status is associated with poor patient outcomes}

Pre-treatment soluble and cellular inflammatory markers were studied and correlated with patient outcomes. Baseline levels of 22 peripheral soluble cytokines/chemokines/ soluble receptors were measured by multiplex assay (see Additional file 2: Table S7 for the panel used) in the peripheral blood of patients and normal donors (ND). irPD patients had elevated baseline plasma levels of CCL4 and CXCL8 chemokines (1042 and 103 pg/mL, respectively) compared to patients with irSD and irPR who had levels similar to ND (131 and $12 \mathrm{pg} / \mathrm{mL}$, Fig. 3a, $p<0.05$ ). No statistically significant differences were detected for other soluble circulating molecules, including CCL3.

Elevated circulating CCL3 (>67 pg/ml), CCL4 (> $600 \mathrm{pg} / \mathrm{ml})$ and CXCL8 $(>76 \mathrm{pg} / \mathrm{ml})$ correlated with poor OS (Hazard Ratio (HR) $=2.8$ (95\%CI:1.17-6.65), $p<0.016$; $\mathrm{HR}=8.4 \quad(95 \% \mathrm{CI}: 2.86-24.44), \quad p<0.0001$ and $\mathrm{HR}=3.5$ (95\%CI:1.43-8.7), $p<0.004$ respectively, Fig. 3b). In patients with an elevation of any of CCL3 or CCL4 or CXCL8, OS was significantly worse $(\mathrm{HR}=2.89$ (95\%CI:1.19-7.05), $p=0.015$, Figure $3 \mathrm{~b}$, bottom right). Pre-treatment levels of CCL3 and CCL4 as continuous variables revealed a statistically predictive significance for OS, HR: 14.14 (95\%CI:1.211-165.25), $p=0.035$ and 5.941 (95\%CI:2.06-17.12), $p=0.001$, respectively (Additional file 2: Table S5). High levels of CCL4 were also associated with levels of $\mathrm{LDH}$ above the upper limit of normal 

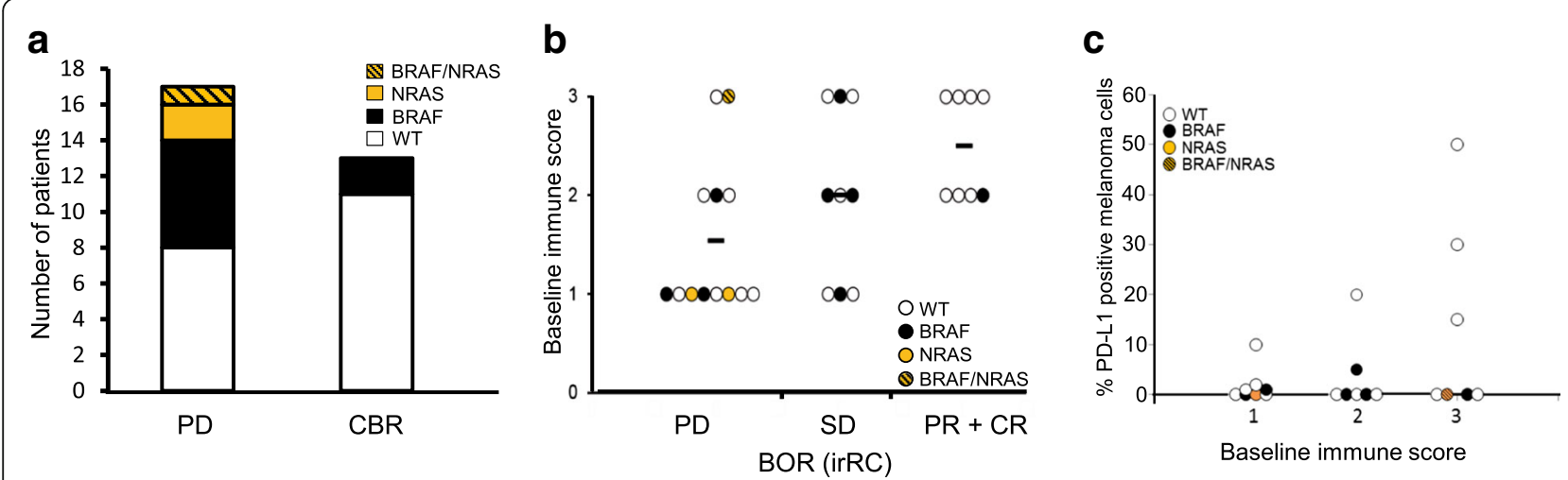

Fig. 2 Intra-tumoral biomarkers linked to clinical outcome. a Number of patients with a BRAF (black), NRAS (orange) or BRAF/NRAS double (orange hatched) mutation in the PD and combined CBR group (CBR; CR, PR or SD $\geq 24$ weeks). The frequency of BRAF and NRAS mutations in PD is significantly higher compared to CBR $\left(X^{2}=4.474, p=0.03\right)$ b Graphical presentation of the baseline immune score (0: absent; 1: low; 2: moderate; 3: high peri- and intratumor CD3- ${ }^{+}$cells) per response group (irRC criteria). Mutations type for each sample is indicated: BRAF (black), NRAS (orange) or BRAF/NRAS double (orange hatched). A significant correlation between response group and baseline immune score was observed (Pearson correlation 0.487, $p=0.01$ ). c Baseline immune score (1: low; 2: moderate; 3 : high peri- and intra-tumor CD3 ${ }^{+}$cells) and the percentage of PD-L1 expressing melanoma cells. Mutations type for each sample is indicated: BRAF (black), NRAS (orange) or BRAF/NRAS double (orange hatched). A trend towards higher PDL1 expression with increasing baseline immune score was observed (Pearson correlation 0.386, $p=0.069$ ). No significant difference between the percentage of PD-L1 positive melanoma cells in tumors with baseline immune score 1 and 3 (Mann Whitney $U$ test $p=0.694$ )

( $p=0.0179$, two-tailed unpaired $\mathrm{t}$ test). The data suggest that elevation of the above-mentioned chemokines reveal a pre-existing state of inflammation for patients that predict a poor outcome to therapy with ipilimumab and CP.

Immunophenotyping of PBMCs pre-study treatment revealed no significant changes in the frequency of major immune cell populations (Additional file 1: Figure S2). Analysis of $\mathrm{T}$ cell subpopulations suggested that objective responders (irPR + irCR) had a lower proportion of circulating activated $\mathrm{CD} 25^{+} \mathrm{CD} 4^{+}$effector T cells $\left(1 \%\right.$ of $\mathrm{CD} 25^{+}$ cells within the effector $\mathrm{CD} 4^{+} \mathrm{T}$ cell gate) compared to patients with irPD, irSD and ND (mean of 3.3\% ( $p=\mathrm{ns}$ ), $4.5 \%(p=0.042)$ and $5.7 \%(p=0.006)$, respectively as shown in Additional file 1: Figure S3C. However, increase in this population had no impact on OS (Additional file 1: Figure S3D, Additional file 2: Table S5). Lower levels of additional activation markers on $\mathrm{CD}^{+}$and $\mathrm{CD}^{+} \mathrm{T}$ cell subsets where seen in responding patients $(C R+P R)$ although not statistically significant (Additional file 1: Figure S3A-B).

B lymphocyte differentiation pattern was assessed from peripheral blood to evaluate pre-existing inflammatory status; more advanced B cell differentiation indicates an ongoing immune response. Indeed, patient response to treatment was associated with their baseline activation pattern of circulating $B$ cells. Circulating $B$ cell subsets from irPD patients displayed a shift towards a late activation/memory phenotype based on surface IgD (sIgD) and CD38 expression [25] (Figure 4a). Specifically, the $\mathrm{Bm} 2$ subset $\left(\operatorname{IgD}^{+} \mathrm{CD} 38^{\text {int }}\right.$, mature resting $\mathrm{B}$ cell) represented $44 \%$ of total $\mathrm{CD} 19^{+}$cells in irPD patients, compared to $64 \%$ in $\operatorname{irPR}+\operatorname{irCR}(p<0.05)$ and $67 \%$ in irSD $(p<0.05)$ patients (ND at $73.9 \%, p<0.01$ when compared to irPD; Figure $4 \mathrm{a})$. The eBm5 + Bm5 subsets (memory B cells, IgD $^{-} \mathrm{CD} 38^{\text {int } / l o}$ ) represented $42.1 \%$ in irPD compared to $19 \%$ in irPR $+\operatorname{irCR}(p<0.05)$ and $19.8 \%$ in $\operatorname{irSD}(p<0.05)$ patients. ND was similar to responders at $18.2 \%$ (Figure $4 \mathrm{a}$ ). Moreover, pre-existing advanced $\mathrm{B}$ cell differentiation had a major impact on OS, where low levels of early differentiated Bm2 $(<57 \%)$ was strongly associated with poor survival: $H R=0.26$ (95\%CI:0.09-0.69), $p=0.004$ (Figure 4b). Conversely, high eBm5 + Bm5 (>14\%) levels were strongly associated with better OS: $\mathrm{HR}=2.65$ (95\%CI:1.07-6.53), $p=0.029$, Figure 4b). An association with OS was also observed when evaluating baseline $\mathrm{Bm} 2$ and $\mathrm{eBm} 5+\mathrm{Bm} 5$ as continuous variables $(\mathrm{HR}=0.113(95 \% \mathrm{CI} \quad 0.023-0.549)$, $p=0.007$ and $\mathrm{HR}=10.27$ (95\%CI:1.30-80.96), $p=0.027$, respectively, Additional file 2: Table S5). These results show that pre-activated B cell differentiation status was associated with poor outcome.

OS was evaluated in patients with favorable B cell differentiation (i.e. high $\mathrm{Bm} 2$ ) combined with low levels of at least one described chemokine. These groups demonstrated highly significant improvements in OS $\left(\mathrm{Bm} 2^{\mathrm{hi}} /\right.$ $\mathrm{CCL}^{\text {lo }} \mathrm{HR}=0.14$ (95\%CI:0.04-0.49) $p=0.0004 ; \mathrm{Bm}^{\text {hi }} /$ $\mathrm{CCL}^{\text {lo }} \quad \mathrm{HR}=0.21 \quad$ (95\%CI:0.08-0.57), $\quad p=0.001$; and $\mathrm{Bm} 2^{\text {hi }} /$ CXCL $^{\text {lo }}{ }^{\text {HR }}=0.29$ (95\%CI:0.11-0.76), $p=0.007$, Figure 5). Finally, patients with high levels of $\mathrm{Bm} 2$ and low levels of all 3 chemokines similarly displayed improved OS (HR $=0.19$ (95\%CI:0.05-0.65), $p=0.003$ ). 


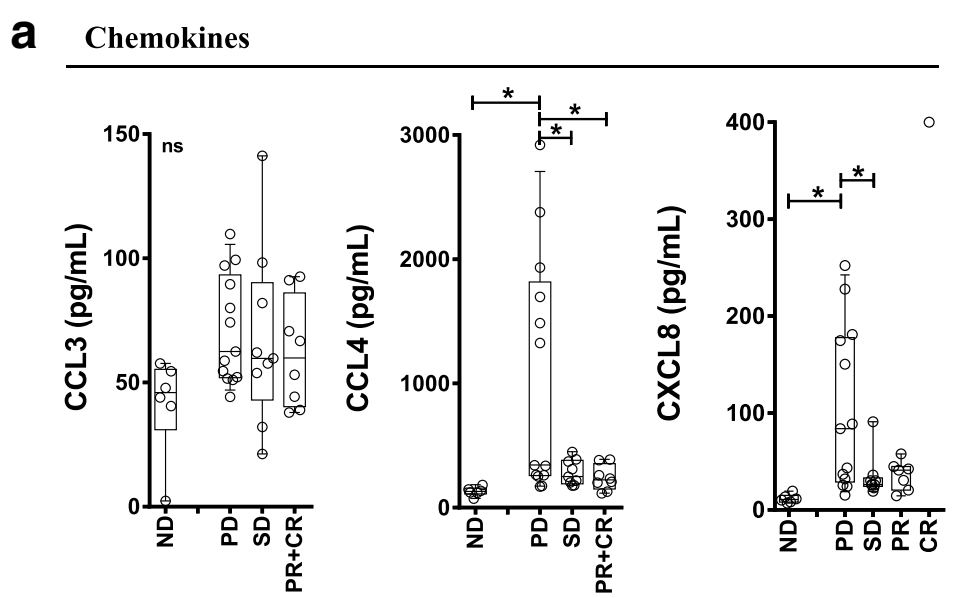

\section{b CCL3}

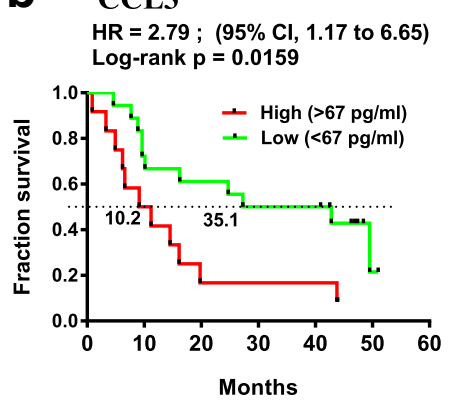

CXCL8

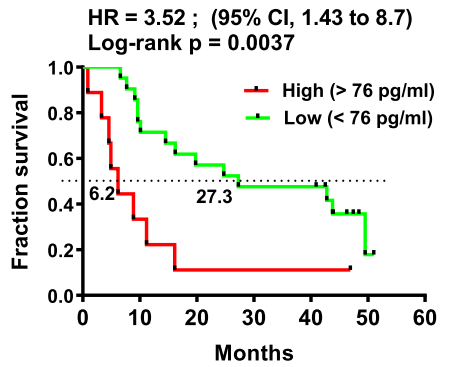

CCL4
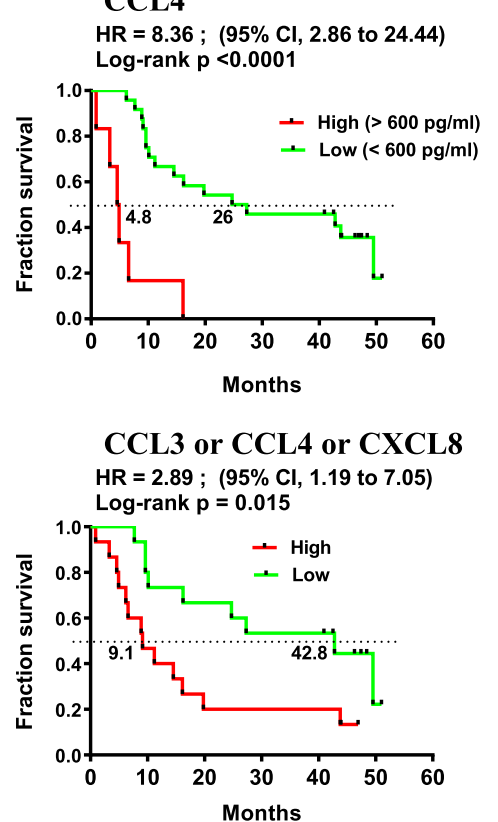

Fig. 3 Pre-treatment peripheral chemokine levels associated with response and OS. a Baseline (pre-treatment) levels of indicated chemokines according to response status. Each point represents a donor, average is outlined, and error bars represent SEM. ns: not significant; *: $p<0.05$ from a one-way ANOVA with Bonferroni post hoc test. b Kaplan-Meier OS curves for the indicated chemokines stratified by levels above (high) or below (low) the mean level for each chemokine. $P$ values were calculated using the log-rank test and are shown on each graph

Overall, data obtained from peripheral markers collected before any treatment show that increase of CCL3, CCL4, CXCL8 or advanced B lymphocyte differentiation status were strongly associated with worse clinical outcomes.

\section{Treatment was associated with changes in B cell} differentiation status and an increase in antibody production B lymphocyte differentiation status and circulating antibody levels were measured throughout the treatment. In both $\mathrm{Bm} 2$ and $\mathrm{eBm} 5+\mathrm{Bm} 5$ subsets, the baseline difference observed in irPR + irCR compared to irPD patients was upheld throughout the study (from a linear mixed effect models analysis, $p<0.05$ for Bm2, $p<0.01$ for $\mathrm{eBm} 5$ and $p=0.058$ for $\mathrm{Bm} 5$ respectively; Figure 6a). Conversely, study treatment was accompanied by a change in B cell subset composition across all patient response groups. The $\mathrm{Bm} 3-4$ subsets $\left(\mathrm{sIgD}^{-} \mathrm{CD} 38^{\text {hi }}\right)$, corresponding to centroblasts/centrocytes germinal center B cells, increased in all patients following initial exposure to ipilimumab $(p<0.05$ for variation in time for all groups, Figure $6 \mathrm{a})$. The $\mathrm{Bm} 3-4$ subset represented over $10 \%$ of B cells at week 10 , compared to $3 \%$ before treatment (Fig. 6a). To investigate whether treatment had 


\section{a B cell differentiation}

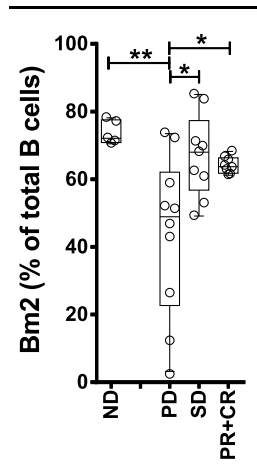

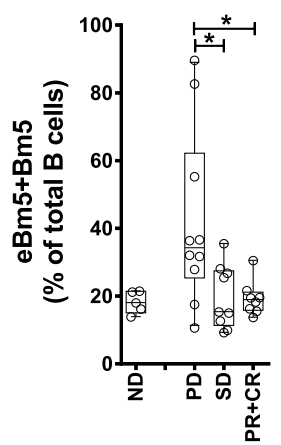

b

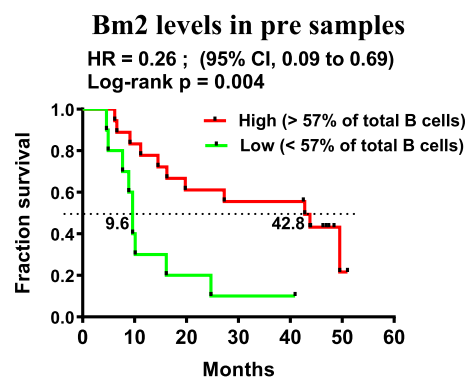

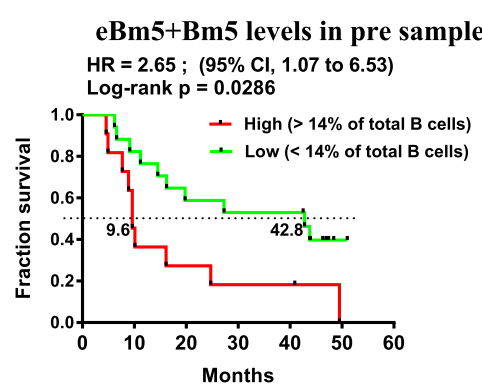

Fig. 4 Pre-treatment circulating B lymphocyte differentiation status associated with patient outcomes. a Baseline (pre-treatment) percentages of indicated B lymphocytes differentiation status (Bm2 and eBm5 + Bm5), according to the Bm classification (slgD and CD38; see Additional file 1: Figure S4A for nomenclature and gating strategy), according to response status. Each point represents a donor, average is outlined, and error bars represent SEM. ns: not significant; * $p<0.05 ;{ }^{* *}: p<0.01$, from a one-way ANOVA with Bonferroni post hoc test. b Kaplan-Meier OS curves for the Bm2 and eBm5 + Bm5 populations, stratified by percentages above (high) or below (low) the mean percentage for each population.

$P$ values were calculated using the log-rank test and are shown on each graph

further influence on B cell activity, levels of circulating immunoglobulins were evaluated. Circulating IgG increased for all patient groups $(p<0.001)$, IgA also increased but was not statistically significant, while IgM levels remained unchanged (Figure $6 \mathrm{~b}$ ).

These results illustrate that B cell phenotype and function were affected in all patients receiving ipilimumab and CP. irPD patients maintained advanced B cell differentiation status throughout treatment.
Ipilimumab exposure is followed by an increase in PD-1+ T cell subsets in non-responders

The levels of selected surface markers related to $\mathrm{T}$ cell function were evaluated on circulating $\mathrm{CD} 4^{+}$and $\mathrm{CD}^{+} \mathrm{T}$ cells before and throughout treatment with ipilimumab and $\mathrm{CP}$. In $\mathrm{CD} 4^{+} \mathrm{T}$ cells, a trend increase in the PD- $1^{+}$cells was observed following exposure to ipilimumab, although this apparent increase did not yield statistically significant correlations with response
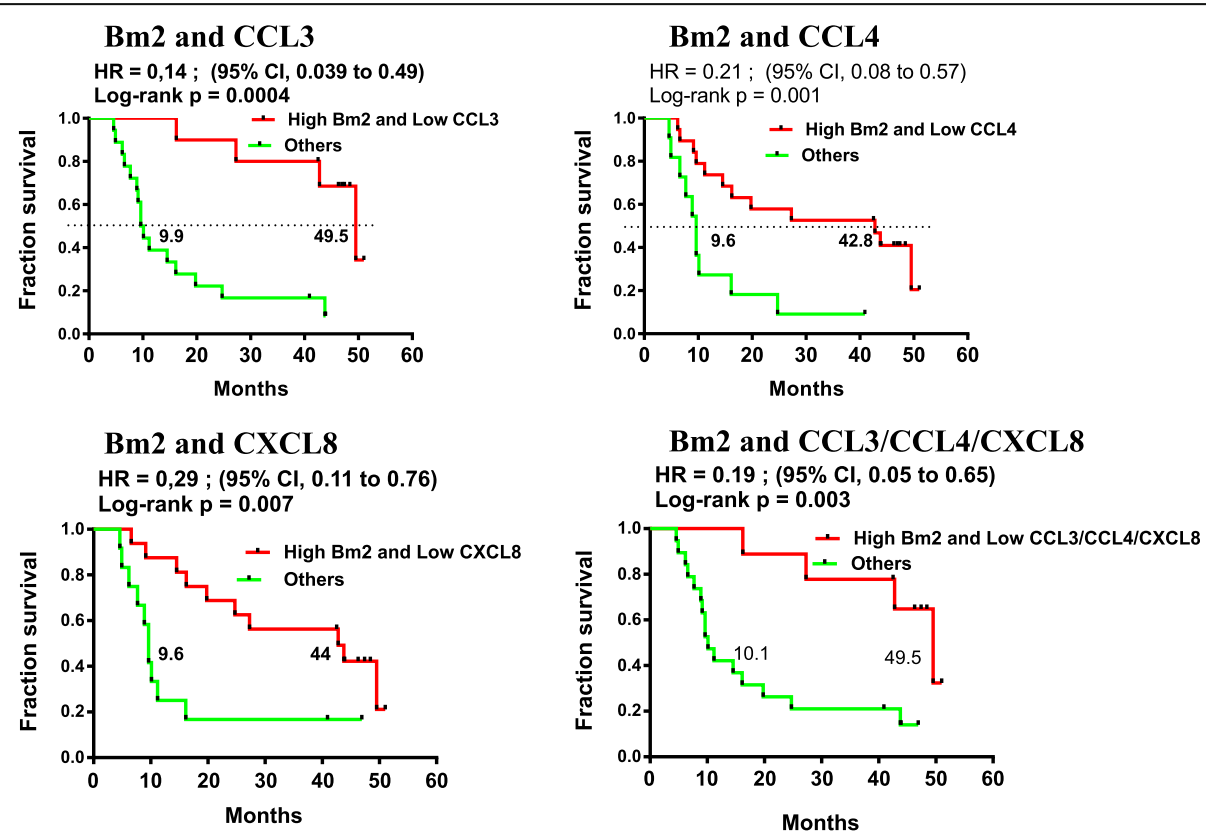

Fig. 5 B cell subsets paired with chemokine levels correlate with OS. Kaplan-Meier OS curves comparing patients with high Bm2 population and low levels of listed chemokine ( $<67 \mathrm{pg} / \mathrm{ml}$ for CCL3, or $<600 \mathrm{pg} / \mathrm{ml}$ for CCL4 or $<76 \mathrm{pg} / \mathrm{ml}$ for CXCL8) versus all other patients (others). $P$ values were calculated using the log-rank test and are shown on each graph 

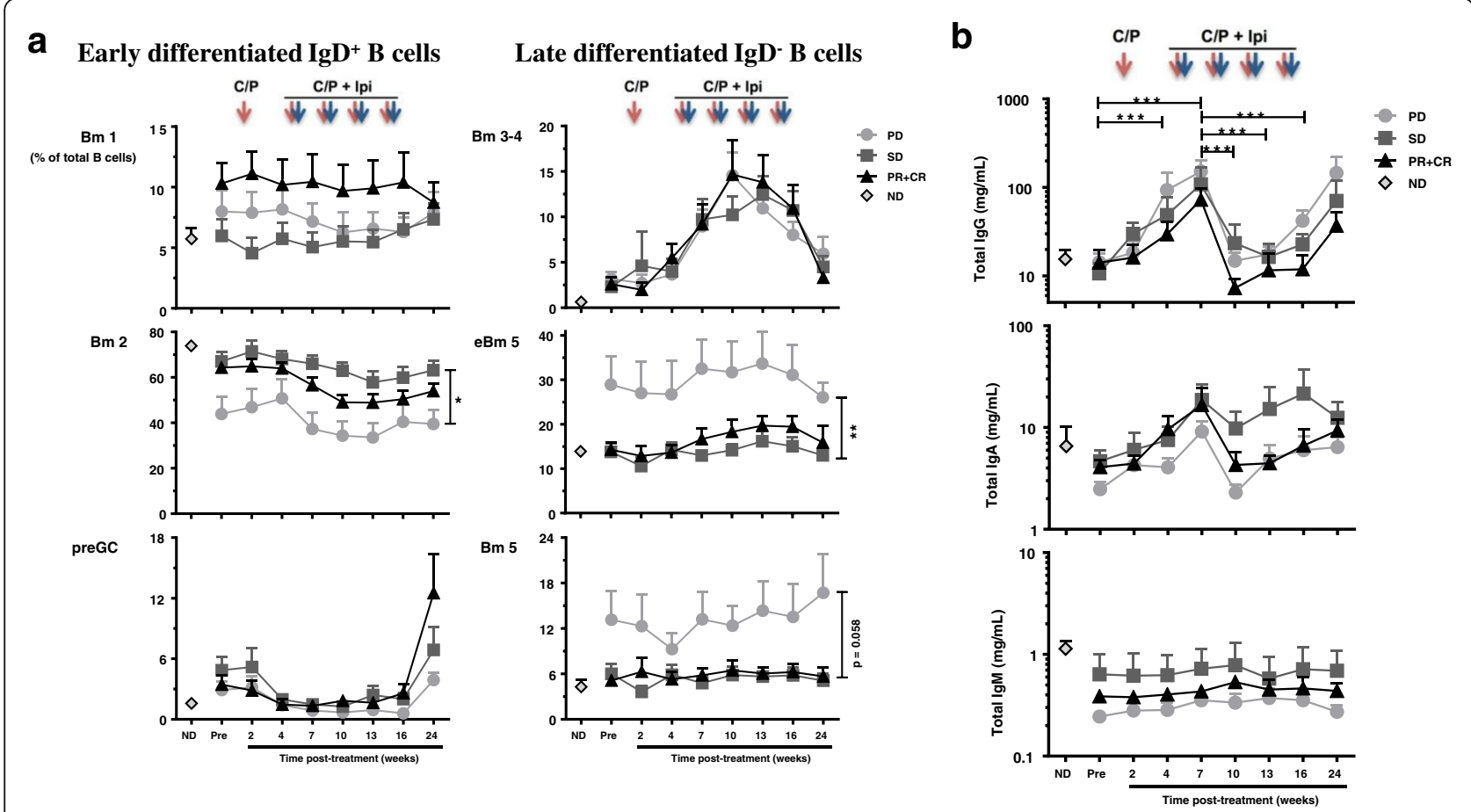

Fig. 6 On treatment monitoring of circulating B lymphocyte differentiation status and Ig levels. a Circulating B lymphocyte differentiation status for each Bm population before and during treatment. Effect of response groups and time points, and their interaction were assessed with a one-way ANOVA group comparison using a linear mixed effects models for repeated measures; between indicated groups, *: $p<0.05$; **: $p<0.01$. $\mathbf{b}$ Evaluations of total circulating levels of lgG (top), IgA (middle) and IgM (bottom). A one-way ANOVA group comparison using a linear mixed effects models for repeated measures statistical analysis reveals that all groups had statistical variations in time for lgG; ${ }^{* * *}: p<0.001$

or survival (Additional file 1: Figure S5A). No discernable increase was observed in $\mathrm{CD}^{+}$Treg cells during treatment (Additional file 1: Figure S5B). Pretreatment proportions of $\mathrm{CD}^{+}$Treg cells did not correlate with response or survival (Additional file 1: Figure S5B, Additional file 2: Table S5). In contrast, increased proportions of $\mathrm{PD}-1^{+} \mathrm{CD} 8^{+} \mathrm{T}$ cells were seen following ipilimumab exposure in patients with irPD $(p<0.05$, Fig. 7). Although baseline proportions of $\mathrm{PD}-1^{+} \mathrm{CD} 8^{+}$cells were not associated with response $(\mathrm{p}=\mathrm{ns})$, responding patients had a significantly lower proportion of $\mathrm{PD}-1^{+} \mathrm{CD}^{+}$cells than patients with irPD at the end of the observation period $(p<0.05$, Figure 7a). There was a trend in association between high levels of $\mathrm{PD}-1^{+} \mathrm{CD}^{+}$cells prior to start of treatment and worse OS (HR $=2.42$ (95\%CI:0.956.20), $p=0.057$, Figure 7b). However, patients with higher proportions of these cells in circulation during treatment consistently had poorer OS $(\mathrm{HR}=3.84$ (95\%CI:1.43-10.31), $p=0.004$ at week 10; $\mathrm{HR}=3.53$ (95\%CI:1.37-9.09), $p=0.005$ at week 13 and $\mathrm{HR}=2.84$ (95\%:1.00-8.05), $p=0.040$ at week 24 (Figure 7c). A similar association between circulating $\mathrm{PD}-1^{+} \mathrm{CD} 8^{+} \mathrm{T}$ cells during treatment as a continuous variable and OS was observed (Additional file 2: Table S5).
ICOS expression was increased uniformly across all patient response groups on both $\mathrm{CD}^{+}$and $\mathrm{CD} 8^{+} \mathrm{T}$ cells following ipilimumab exposure, as previously observed for ipilimumab monotherapy (Additional file 1: Figure S6; top panels [7, 26, 27]). Increase in ICOS was not associated with response or OS (Additional file 1: Figure S6; lower panels, Additional file 2: Table S5).

\section{Discussion}

This phase II study explored the toxicity, activity and predictive biomarkers of the combination of $\mathrm{CP}$ with ipilimumab. The study of this combination reveals manageable toxicity, with most patients completing the full course of treatment. IrAEs were consistent with previously published studies [2, 28]. Our study showed fewer grade 3/4 irAEs and no immune-related hepatotoxicity, when compared to ipilimumab-DTIC arm, where more than $33 \%$ of patients experienced grade 3 or more irAEs [3]. The difference in toxicity profile may be explained by the lower dose of ipilimumab used in this study or the use of prophylactic corticosteroids prior to paclitaxel infusions, thus potentially diminishing the severity of irAEs. A previous study showed higher hepatotoxicity when ipilimumab was combined with DTIC, rather than carboplatin/paclitaxel [28]. The performed comprehensive immune 


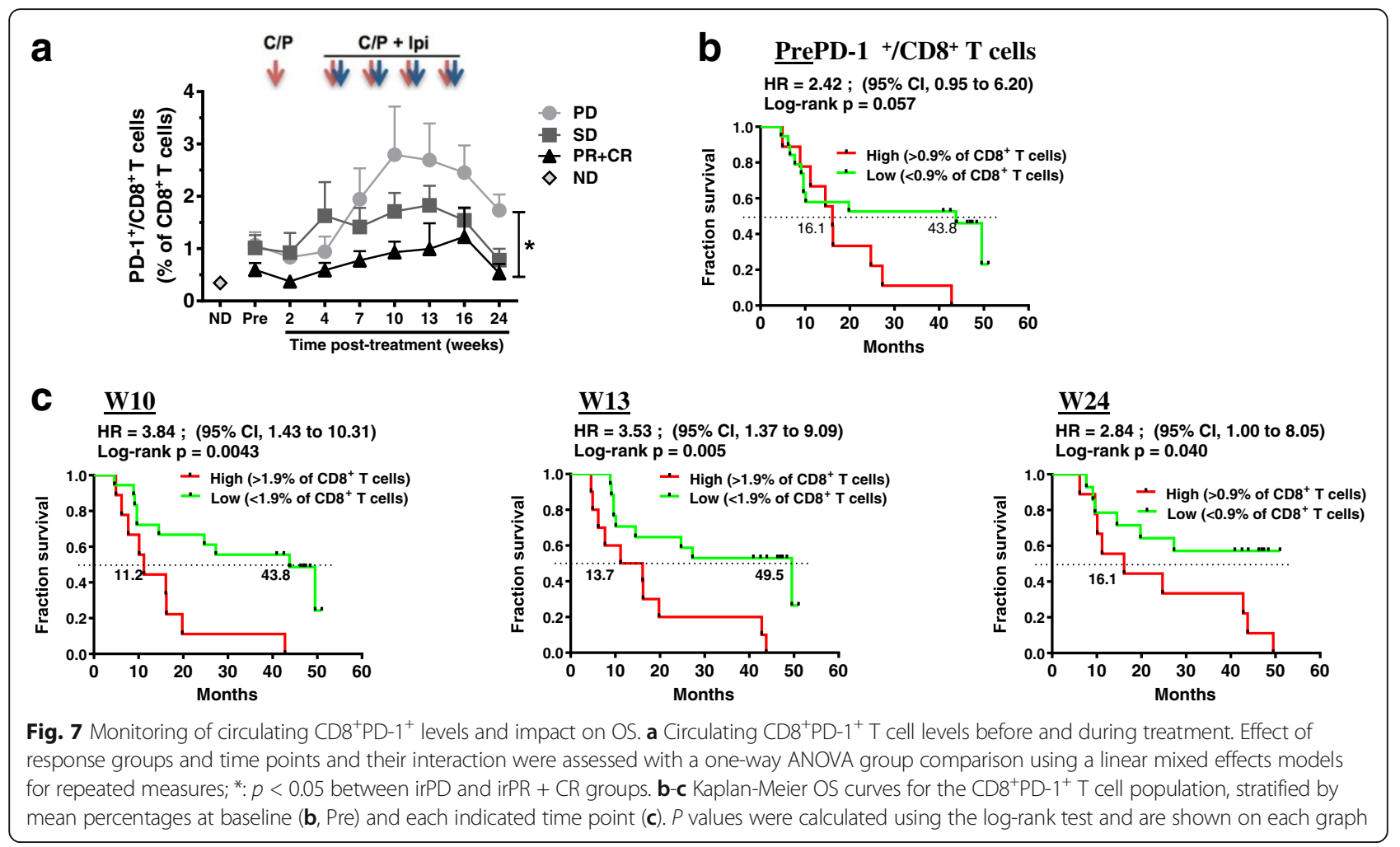

correlative study revealed new predictive circulating signatures linked to pre-existing inflammatory state associated with worse patient outcome.

The median OS (16.2 months) as well as 1-year OS (56.7\%), 2-year OS (43.3\%) and 3-year OS (36.7\%) were comparable to previously published responses to ipilimumab in the first-line setting yet this regimen offers no survival benefit [3, 29-32]. Similarly to Weber et al. the observed ORR was $27 \%$ by irRC criteria [28]. The limitations of cross-study comparisons are recognized. Outcomes from the current study may have been influenced by the small patient population with mostly normal pretreatment LDH, few patients with brain metastases and most patients with ECOG of 0 . The criteria used to assess response (irRC versus RECIST 1.1) and the availability of additional systemic treatment options postprogression may have affected the results.

The impact of prophylactic corticosteroids use on anti-tumor immune responses with ipilimumab is poorly understood and a source of concern. Sequential treatment with ipilimumab and $\mathrm{CP}$ previously showed improved progression-free survival (PFS) in non-small cell lung carcinoma compared to concurrent treatment in a randomized phase II trial [33]. Patients on this current study were randomized in a 1:2 fashion favoring the sequential arm. Our results in melanoma suggest effective anti-tumor activity regardless of the scheduling of corticosteroid premedication (Additional file 2: Table S3).
Understanding mechanisms that influence patient response to immunotherapies will help shape future targeting strategies of the immune response. Aspects of the immune response were studied both systemically and within the tumor environment. The genetic composition of the tumor may ultimately impact on its immunogenicity. Although there is evidence that mutant BRAF could trigger immune responses in melanoma cells under certain contexts [34], our data are more consistent with a diminished chance of clinical benefit to ipilimumab in patients with BRAF mutations [35-37]. Recent subgroup analysis results from the CheckMate 067 demonstrated a favorable OS outcome for patients with $B R A F$-mutant tumors in the ipilimumab cohort in previously untreated patients [30]. 6/9 BRAF mutated patients had failed a BRAF inhibitor which may have contributed to poorer outcomes. One report demonstrated that patients with NRAS mutant metastatic melanoma achieved increased clinical benefit from ipilimumab compared to patients with $B R A F / N R A S$ wild type melanomas [38]. We did not confirm these results in our study, since our three patients with NRAS mutant melanoma all had PD as BOR.

A higher immune score in pre-treatment tumor biopsies correlated with BOR. This correlation is in line with the predictive value of $\mathrm{T}$ cell infiltrates in regional nodal metastases and benefit in patients treated with neoadjuvant interferon- $\alpha-2 b$ therapy $[39,40]$. The presence of a 
strong immune cell infiltrate in the tumor microenvironment suggests that the tumor elicits $\mathrm{T}$ cell infiltration and that anti-tumor $\mathrm{T}$ cell activation can be enhanced by stimulating signals such as ipilimumab.

In contrast, we found that increased systemic inflammation at baseline is associated with poor response to therapy and poor OS. Non-responders displayed baseline differences in circulating chemokines CXCL8 and CCL4 in comparison to the levels of NDs and responders. CXCL8 is associated with chronic inflammatory states [41]. Moreover, circulating levels of CXCL8 have previously been associated with poorer prognosis in melanoma and other malignancies [42, 43], and an early increase in serum CXCL8 after initiation of anti-PD-1 treatment was associated with poor response and survival [44]. CCL4 and CCL3 may mediate the recruitment of $\mathrm{CD}^{+} \mathrm{T}$ cells and regulatory $\mathrm{T}$ cells within melanoma lesions [45]. In this trial, baseline elevated circulating CCL3, CCL4 and CXCL8, alone or in combination, were associated with substantially worse patient outcomes (Figs. 3 and 5), thus making them potential predictive biomarkers for patients most likely to fail treatment. The role of chemokines in mediating resistance to therapy remains to be investigated.

A specific skewing in B cell differentiation prior to treatment was noted in non-responders, with higher eBm5/Bm5 terminally differentiated B cells compared to responders and NDs (Figure 4). This also strongly correlated with OS. These results may reflect a general state of inflammation in non-responders as reported in other chronic conditions [46, 47]. B cell differentiation status was maintained throughout the study, suggesting that ipilimumab did not reverse this effect. Interestingly, Bm3-4 population, corresponding to centroblasts/centrocytes antibody producing B cells, increased with treatment in all patients, independently of clinical outcome. Study treatment had an effect on B cell biology, leading to fluctuations in circulating antibodies. CTLA-4 has been shown to modulate B cell responses through modulation of $\mathrm{T}$ follicular helper, $\mathrm{T}$ follicular regulatory, and $\mathrm{T}$ regulatory cells in animal models $[48,49]$. This is the first evidence showing that ipilimumab treatment in humans affects B lymphocyte differentiation and function. However, the contribution of chemotherapy and the influence of systemic corticosteroids as prophylaxis remains to be established. The impact of the treatment regimen on OS of patients depended on the baseline B cell differentiation status, alone or in combination with chemokines. The impressive combined effect of baseline early B cell populations and chemokines on OS needs to be validated. Indeed, if these associations are confirmed, they might bring new predictive circulating biomarkers to immunotherapy.
Finally, an increase in the $\mathrm{CD} 8^{+} \mathrm{PD}-1^{+} \mathrm{T}$ cell population in non-responders and a robust impact on OS during treatment could be a sign of immune exhaustion and points to a possible escape mechanism to anti-CTLA-4. It is likely that simultaneous targeting of multiple immune checkpoints such as PD-1 and CTLA-4 [50] may be required to overcome compensatory mechanisms. This further suggests that additional negative immune regulators should be monitored after immunotherapies to identify relevant new targets to overcome resistance mechanisms.

\section{Conclusions}

In summary, we report that ipilimumab and CP can be used in combination yielding manageable toxicities and favorable response, even if given concurrently. Our study revealed tumor-related and peripheral signatures associated with ipilimumab/CP resistance and OS. $\mathrm{CD}^{+} \mathrm{T}$ cell infiltration of the tumor correlated with good response, whereas the presence of a $B R A F$ or NRAS mutation correlated with poor response, especially in patients pretreated with a BRAF inhibitor. Resistance to treatment was associated with preexisting systemic inflammatory state, specifically elevated CCL4 and CXCL8, baseline B lymphocyte subset skewing, and increased $\mathrm{CD} 8^{+} \mathrm{PD}-1^{+} \mathrm{T}$ lymphocytes. From a comprehensive basic immune monitoring, we provide evidence for new predictive circulating biomarkers linked to OS. Larger studies are required to validate these circulating biomarkers. Future studies will address the safety of other checkpoint inhibitors combined with chemotherapy and determine whether biomarkers associated with improved outcomes to CTLA-4 directed therapy may be relevant to the clinical activity of inhibitors of other checkpoints.

\section{Additional files}

Additional file 1: Supplemental Figures. Figure S1. KaplanMeier curves. Figure S2. Evaluation of main immune cell population from samples collected before treatment (Pre). Figure S3. Evaluation of cell surface activation markers on CD4+ and CD8+ T lymphocyte subsets. Figure S4. Bm classification of B cell subsets. Figure S5. T cell subsets $\left(C D 4^{+}\right.$PD $-1^{+}$and Tregs) analysis throughout treatment. Figure S6. ICOS expression on T cells throughout treatment. (PPTX $653 \mathrm{~kb}$ )

Additional file 2: Supplemental Tables. Table S1. Demographic and baseline characteristics of the patients seperated by arm. Table S2. Adverse Events. Table S3. Tumor Response (by irRC, mWHO and Arm).

Table S4. PD-L1 expression in the two Best Overall Response groups. Table S5. Univariate Cox regression models. Table S6. Antibodies for characterization of circulating immune cells. Table S7. Peripheral soluble cytokines/chemokines/soluble receptors studied by multiplex. (DOCX 50 kb)

\section{Abbreviations}

AE: Adverse event; BOR: Best overall response; BORR: Best overall response rate; CBR: Clinical benefit rate; CP: Carboplatin paclitaxel; CR: Complete response; CTLA-4: Cytotoxic T-cell lymphocyte-associated protein 4; DCR: Disease control rate; ECOG: Eastern cooperative oncology group; irAE: Immune-related adverse event; irRC: Immune-related response criteria; LDH: Lactate dehydrogenase; MAPK: Mitogen-activated protein kinase; MDSC: Myeloid-derived suppressor cells; mWHO: Modified World Health Organization; ND: Normal donor; OS: Overall survival; PD: Progressive disease; 
PD-L1: Programmed death-ligand 1; PFS: Progression free survival; PR: Partial response; SAE: Serious adverse event; SD: Stable disease

\section{Acknowledgements}

The authors would like to acknowledge Martin Ladouceur from the Biostatistics platform of the CRCHUM for providing statistical analyses on the peripheral blood correlative studies. The authors would also like to thank the patients for their participation, and the staff at each site.

\section{Funding}

This work was conducted with research funds from Bristol-Myers Squibb and the Rossy Cancer Network.

\section{Availability of data and materials}

The datasets used and/or analyzed during the current study available from the corresponding author on reasonable request.

\section{Authors' contributions}

RJ, RL, EC, LCVK, AS, WHM conceptualized and designed the trial. RJ, SK, JEF, $J D, K B, J-P A, C L, A S, W H M$ participated in the enrollment and care of patients on trial. PT, RL, J-FC, LCVK, AS directed the collection and analysis of immune correlates. RJ, RL, EC, PT, SK, CL, HL, LCVK, AS, WHM were responsible for data collection and analysis. RJ, RL, EC, PT, CL, JE-H, LCVK, WHM wrote the manuscript. All authors read and approved the final manuscript.

\section{Ethics approval and consent to participate}

This study was reviewed and approved by the Institutional Review Board at the Jewish General Hospital and Hopital Notre-Dame. Written informed consent was obtained from the patients prior to treatment.

\section{Consent for publication}

Written informed consent was obtained from the patients for publication of their individual details and accompanying images in this manuscript. The consent form is held by the authors and is available for review by the Editor-in-Chief.

\section{Competing interests}

R Jamal is an advisor and has received honoraria from Bristol-Myers Squibb. WH Miller Jr. is an advisor and has received honoraria from Bristol-Myers Squibb.

\section{Publisher's Note}

Springer Nature remains neutral with regard to jurisdictional claims in published maps and institutional affiliations.

\section{Author details \\ 'Hôpital Notre-Dame, Centre de recherche du CHUM, Centre hospitalier de I'Université de Montréal, Montréal, QC, Canada. ${ }^{2}$ Centre de recherche du CHUM, Institut du Cancer de Montréal, Université de Montréal, Montréal, QC, Canada. ${ }^{3}$ Segal Cancer Center, Jewish General Hospital, Rossy Cancer Network, McGill University, 3755 Côte-St-Catherine, suite E670, Montreal, Québec, Canada. ${ }^{4}$ Department of Pathology, Molecular Pathology Center, Jewish General Hospital, McGill University, Montreal, QC, Canada.}

Received: 9 June 2017 Accepted: 4 October 2017

Published online: 21 November 2017

\section{References}

1. Korn EL, Liu PY, Lee SJ, Chapman JA, Niedzwiecki D, Suman VJ, et al. Metaanalysis of phase II cooperative group trials in metastatic stage IV melanoma to determine progression-free and overall survival benchmarks for future phase II trials. J Clin Oncol. 2008;26:527-34.

2. Hodi FS, O'Day SJ, McDermott DF, Weber RW, Sosman JA, Haanen JB, et al. Improved survival with ipilimumab in patients with metastatic melanoma. N Engl J Med. 2010;363:711-23.

3. Robert C, Thomas L, Bondarenko I, O'Day S, Weber J, Garbe C, et al. Ipilimumab plus dacarbazine for previously untreated metastatic melanoma. N Engl J Med. 2011;364:2517-26.

4. Theurich S, Rothschild SI, Hoffmann M, Fabri M, Sommer A, Garcia-Marquez $M$, et al. Local tumor treatment in combination with systemic Ipilimumab immunotherapy prolongs overall survival in patients with advanced malignant melanoma. Cancer Immunol Res. 2016;4:744-54.
5. Kang J, Demaria S, Formenti S. Current clinical trials testing the combination of immunotherapy with radiotherapy. J Immunother Cancer. 2016;4:51.

6. Reck M, Luft A, Szczesna A, Havel L, Kim SW, Akerley W, et al. Phase III Randomized Trial of Ipilimumab Plus Etoposide and Platinum Versus Placebo Plus Etoposide and Platinum in Extensive-Stage Small-Cell Lung Cancer. J Clin Oncol 2016;34:3740-748.

7. Wang W, Yu D, Sarnaik AA, Yu B, Hall M, Morelli D, et al. Biomarkers on melanoma patient T cells associated with ipilimumab treatment. J Transl Med. 2012;10:146

8. Patel SP, Kim DW, Bassett RL, Cain S, Washington E, Hwu WJ, et al. A phase II study of ipilimumab plus temozolomide in patients with metastatic melanoma. Cancer Immunol Immunother. 2017;66:1359-366.

9. Roh W, Chen PL, Reuben A, Spencer CN, Prieto PA, Miller JP, et al. Integrated molecular analysis of tumor biopsies on sequential CTLA-4 and PD-1 blockade reveals markers of response and resistance. Sci Transl Med. 2017;9

10. Zitvogel L, Kroemer G. Anticancer immunochemotherapy using adjuvants with direct cytotoxic effects. J Clin Invest. 2009;119:2127-30.

11. Tesniere A, Schlemmer F, Boige V, Kepp O, Martins I, Ghiringhelli F, et al. Immunogenic death of colon cancer cells treated with oxaliplatin. Oncogene. 2010;29:482-91.

12. Inoue $\mathrm{H}$, Tani K. Multimodal immunogenic cancer cell death as a consequence of anticancer cytotoxic treatments. Cell Death Differ. 2014:21:39-49.

13. Merritt RE, Mahtabifard A, Yamada RE, Crystal RG, Korst RJ. Cisplatin augments cytotoxic T-lymphocyte-mediated antitumor immunity in poorly immunogenic murine lung cancer. J Thorac Cardiovasc Surg. 2003;126:1609-17.

14. Correale P, Del Vecchio MT, La Placa M, Montagnani F, Di Genova G, Savellini GG, et al. Chemotherapeutic drugs may be used to enhance the killing efficacy of human tumor antigen peptide-specific CTLS. J Immunother. 2008:31:132-47.

15. Hodge JW, Garnett CT, Farsaci B, Palena C, Tsang KY, Ferrone S, et al. Chemotherapy-induced immunogenic modulation of tumor cells enhances killing by cytotoxic T lymphocytes and is distinct from immunogenic cell death. Int J Cancer. 2013;133:624-36.

16. Sevko A, Michels T, Vrohlings M, Umansky L, Beckhove P, Kato M, et al. Antitumor effect of paclitaxel is mediated by inhibition of myeloid-derived suppressor cells and chronic inflammation in the spontaneous melanoma model. J Immunol. 2013:190:2464-71.

17. Jure-Kunkel MN, Masters G, Girit E, Dito G, Lee FY. Antitumor activity of antiCTLA-4 monoclonal antibody (mAb) in combination with ixabepilone in preclinical tumor models. J Clin Oncol. 2008;26(20 suppl). Abstract 3048

18. Wolchok JD, Hoos A, O'Day S, Weber JS, Hamid O, Lebbe C, et al. Guidelines for the evaluation of immune therapy activity in solid tumors: immunerelated response criteria. Clin Cancer Res. 2009;15:7412-20.

19. Altman DG, McShane LM, Sauerbrei W, Taube SE. Reporting recommendations for tumor marker prognostic studies (REMARK): explanation and elaboration. PLoS Med. 2012;9:e1001216.

20. Topalian SL, Hodi FS, Brahmer JR, Gettinger SN, Smith DC, McDermott DF, et al. Safety, activity, and immune correlates of anti-PD-1 antibody in cancer N Engl J Med. 2012;366:2443-54.

21. Taube JM, Klein A, Brahmer JR, Xu H, Pan X, Kim JH, et al. Association of PD-1, PD-1 ligands, and other features of the tumor immune microenvironment with response to anti-PD-1 therapy. Clin Cancer Res. 2014;20:5064-74.

22. Grange C, Letourneau J, Forget MA, Godin-Ethier J, Martin J, Liberman M, et al. Phenotypic characterization and functional analysis of human tumor immune infiltration after mechanical and enzymatic disaggregation. J Immunol Methods. 2011;372:119-26.

23. Godin-Ethier J, Pelletier S, Hanafi LA, Gannon PO, Forget MA, Routy JP, et al. Human activated T lymphocytes modulate IDO expression in tumors through Th1/Th2 balance. J Immunol. 2009;183:7752-60.

24. Team RC. R: a language and environment for statistical computing. 2008.

25. Bohnhorst JO, Bjorgan MB, Thoen JE, Natvig JB, Thompson KM. Bm1-Bm5 classification of peripheral blood $B$ cells reveals circulating germinal center founder cells in healthy individuals and disturbance in the B cell subpopulations in patients with primary Sjogren's syndrome. J Immunol. 2001;167:3610-8.

26. Liakou Cl, Kamat A, Tang DN, Chen H, Sun J, Troncoso P, et al. CTLA-4 blockade increases IFNgamma-producing CD4+ICOShi cells to shift the ratio of effector to regulatory T cells in cancer patients. Proc Natl Acad Sci U S A. 2008;105:14987-92. 
27. Chen H, Liakou Cl, Kamat A, Pettaway C, Ward JF, Tang DN, et al. Anti-CTLA4 therapy results in higher CD4+ICOShi T cell frequency and IFN-gamma levels in both nonmalignant and malignant prostate tissues. Proc Natl Acad Sci U S A. 2009:106:2729-34.

28. Weber J, Hamid O, Amin A, O'Day S, Masson E, Goldberg SM, et al. Randomized phase I pharmacokinetic study of ipilimumab with or without one of two different chemotherapy regimens in patients with untreated advanced melanoma. Cancer Immun. 2013;13:7.

29. Robert C, Schachter J, Long GV, Arance A, Grob JJ, Mortier L, et al. Pembrolizumab versus Ipilimumab in Advanced Melanoma. N Engl J Med. 2015;372:2521-32

30. Wolchok JD, Chiarion-Sileni V, Gonzalez R, Rutkowski P, Grob JJ, Cowey CL, et al. Overall Survival with Combined Nivolumab and Ipilimumab in Advanced Melanoma. N Engl J Med. 2017

31. Maio M, Grob JJ, Aamdal S, Bondarenko I, Robert C, Thomas L, et al. Fiveyear survival rates for treatment-naive patients with advanced melanoma who received ipilimumab plus dacarbazine in a phase III trial. J Clin Oncol. 2015;33:1191-6.

32. Schadendorf D, Hodi FS, Robert C, Weber JS, Margolin K, Hamid O, et al. Pooled analysis of long-term survival data from phase II and phase III trials of Ipilimumab in Unresectable or metastatic melanoma. J Clin Oncol. 2015; 33:1889-94.

33. Lynch TJ, Bondarenko I, Luft A, Serwatowski P, Barlesi F, Chacko R, et al. Ipilimumab in combination with paclitaxel and carboplatin as first-line treatment in stage IIIB/IV non-small-cell lung cancer: results from a randomized, double-blind, multicenter phase II study. J Clin Oncol. 2012;30: 2046-54.

34. Ilieva KM, Correa I, Josephs DH, Karagiannis P, Egbuniwe IU, Cafferkey MJ, et al. Effects of BRAF mutations and BRAF inhibition on immune responses to melanoma. Mol Cancer Ther. 2014;13:2769-83.

35. Sumimoto $H$, Imabayashi F, Iwata T, Kawakami Y. The BRAF-MAPK signaling pathway is essential for cancer-immune evasion in human melanoma cells. J Exp Med. 2006;203:1651-6.

36. Khalili JS, Liu S, Rodriguez-Cruz TG, Whittington M, Wardell S, Liu C, et al. Oncogenic BRAF(V600E) promotes stromal cell-mediated immunosuppression via induction of interleukin-1 in melanoma. Clin Cancer Res. 2012;18:5329-40.

37. Sapkota B, Hill CE, Pollack BP. Vemurafenib enhances MHC induction in BRAF homozygous melanoma cells. Oncoimmunology. 2013;2:e22890.

38. Johnson DB, Lovly CM, Flavin M, Panageas KS, Ayers GD, Zhao Z, et al. Impact of NRAS mutations for patients with advanced melanoma treated with immune therapies. Cancer Immunol Res. 2015;3:288-95.

39. Mihm MC Jr, Clemente CG, Cascinelli N. Tumor infiltrating lymphocytes in lymph node melanoma metastases: a histopathologic prognostic indicator and an expression of local immune response. Lab Invest. 1996;74:43-7.

40. Moschos SJ, Edington HD, Land SR, Rao UN, Jukic D, Shipe-Spotloe J, et al. Neoadjuvant treatment of regional stage IIIB melanoma with high-dose interferon alfa-2b induces objective tumor regression in association with modulation of tumor infiltrating host cellular immune responses. J Clin Oncol. 2006;24:3164-71.

41. Palomino DC, Marti LC. Chemokines and immunity. Einstein (Sao Paulo). 2015;13:469-73.

42. Sanmamed MF, Carranza-Rua O, Alfaro C, Onate C, Martin-Algarra S, Perez G, et al. Serum interleukin-8 reflects tumor burden and treatment response across malignancies of multiple tissue origins. Clin Cancer Res. 2014;20:5697-707.

43. Fujita Y, Okamoto M, Goda H, Tano T, Nakashiro K, Sugita A, et al. Prognostic significance of interleukin-8 and CD163-positive cell-infiltration in tumor tissues in patients with oral squamous cell carcinoma. PLoS One. 2014;9:e110378

44. Sanmamed MF, Perez-Gracia JL, Schalper KA, Fusco JP, Gonzalez A, Rodriguez-Ruiz ME, et al. Changes in serum interleukin-8 (IL-8) levels reflect and predict response to anti-PD-1 treatment in melanoma and non-smallcell lung cancer patients. Ann Oncol. 2017;28:1988-95.

45. Schlecker E, Stojanovic A, Eisen C, Quack C, Falk CS, Umansky V, et al. Tumor-infiltrating monocytic myeloid-derived suppressor cells mediate CCR5-dependent recruitment of regulatory T cells favoring tumor growth. J Immunol. 2012;189:5602-11.

46. Sugalski JM, Rodriguez B, Moir S, Anthony DD. Peripheral blood B cell subset skewing is associated with altered cell cycling and intrinsic resistance to apoptosis and reflects a state of immune activation in chronic hepatitis $C$ virus infection. J Immunol. 2010;185:3019-27.
47. Moir S, Ho J, Malaspina A, Wang W, DiPoto AC, O'Shea MA, et al. Evidence for HIV-associated B cell exhaustion in a dysfunctional memory B cell compartment in HIV-infected viremic individuals. J Exp Med. 2008;205:1797-805.

48. Sage PT, Paterson AM, Lovitch SB, Sharpe AH. The coinhibitory receptor CTLA-4 controls B cell responses by modulating T follicular helper, $T$ follicular regulatory, and T regulatory cells. Immunity. 2014;41:1026-39.

49. Wing JB, Ise W, Kurosaki T, Sakaguchi S. Regulatory T cells control antigenspecific expansion of Tfh cell number and humoral immune responses via the coreceptor CTLA-4. Immunity. 2014;41:1013-25.

50. Larkin J, Chiarion-Sileni V, Gonzalez R, Grob JJ, Cowey CL, Lao CD, et al. Combined Nivolumab and Ipilimumab or Monotherapy in untreated melanoma. N Engl J Med. 2015;373:23-34

\section{Submit your next manuscript to BioMed Central and we will help you at every step:}

- We accept pre-submission inquiries

- Our selector tool helps you to find the most relevant journal

- We provide round the clock customer support

- Convenient online submission

- Thorough peer review

- Inclusion in PubMed and all major indexing services

- Maximum visibility for your research

Submit your manuscript at www.biomedcentral.com/submit 